\title{
Shape Control for Experimental Continuation
}

\author{
Robin M. Neville, Rainer M. J. Groh, Alberto Pirrera, and Mark Schenk \\ Bristol Composites Institute (ACCIS), Department of Aerospace Engineering, University of Bristol, \\ Bristol BS8 1TR, United Kingdom
}

(Received 5 March 2018; revised manuscript received 20 April 2018; published 21 June 2018)

\begin{abstract}
An experimental method has been developed to locate unstable equilibria of nonlinear structures quasistatically. The technique involves loading a structure by the application of either a force or a displacement at a main actuation point while simultaneously controlling the overall shape using additional bidirectional probe points. The method is applied to a shallow arch, and unstable segments of its equilibrium path are identified experimentally for the first time. Shape control is a fundamental building block for the experimental—as opposed to numerical — continuation of nonlinear structures, which will significantly expand our ability to measure their mechanical response.
\end{abstract}

DOI: 10.1103/PhysRevLett.120.254101

Background.-The force-displacement response of nonlinear structures can be complex and even chaotic. Limit and branch points can partition equilibrium manifolds into stable and unstable segments. In particular, displacement and force limit points change the stability of a structure and inject unstable eigenmodes into the deformation shape, thereby rendering certain segments of the ensuing forcedisplacement manifolds inaccessible experimentally. This kind of behavior is observed even in simple structures, such as the shallow arch studied in this Letter.

Figure 1 shows how force limit points cause forcecontrolled structures to snap to the next available stable equilibrium. Similarly, displacement limit points cause displacement-controlled structures to snap while conserving the displacement at the point(s) of actuation. To trace an equilibrium manifold like that shown in Fig. 1, a means of controlling both the forces acting on the structure and its global deformation is required. This combination is readily implemented in a numerical setting, because force and deformation can be independently controlled via a third parameter, namely, the arclength [1]. However, tracing similar equilibrium manifolds experimentally remains an open challenge. The challenge is that the force and displacement at the actuation point(s) are not independent but rather inherently linked through elasticity. A force applied at a specific control point results in a displacement at that point; similarly, an applied displacement induces a reaction force. This differentiates quasistatic from dynamic problems [2]

Published by the American Physical Society under the terms of the Creative Commons Attribution 4.0 International license. Further distribution of this work must maintain attribution to the author(s) and the published article's title, journal citation, and DOI. where the input vibration frequency and amplitude are decoupled.

In this Letter, we present a general method to explore the unstable equilibria of a nonlinear structure quasistatically. The key to this technique is decoupling force and displacement at a specific control point by introducing a third control variable: the shape of the structure.

Consider a shallow arch loaded transversely at its midpoint as shown in Fig. 2(a), which is a known benchmark for numerical arclength solvers. For given combinations of geometry and material parameters, the arch features particularly pronounced nonlinear behavior $[3,4]$ with many unstable loops in force-displacement space [Fig. 3(a)]. These loops give rise to the problemaccessing unstable equilibria and tracing experimentally

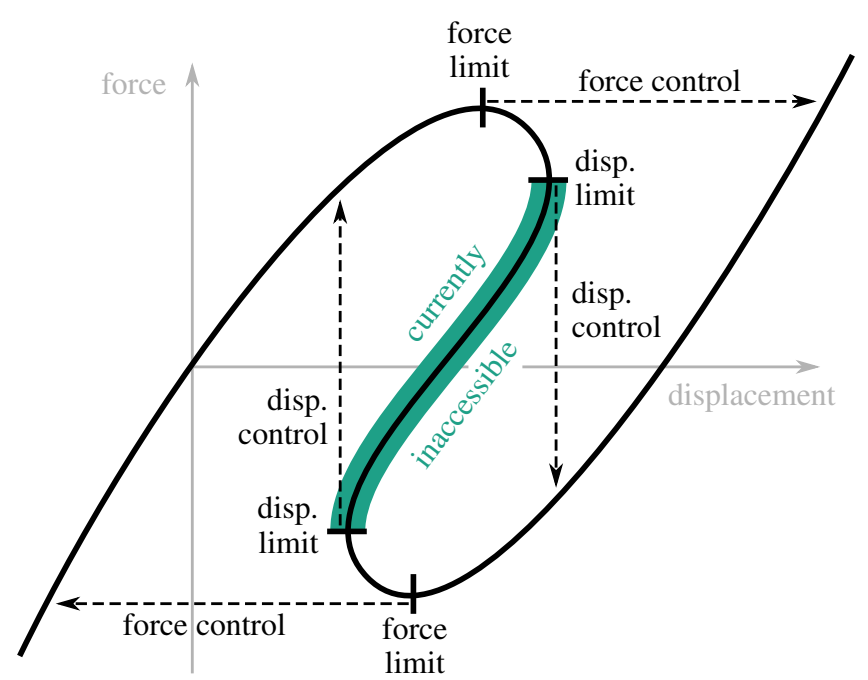

FIG. 1. Limit points cause displacement and force control to snap to different parts of the equilibrium curve, resulting in an experimentally inaccessible region. 

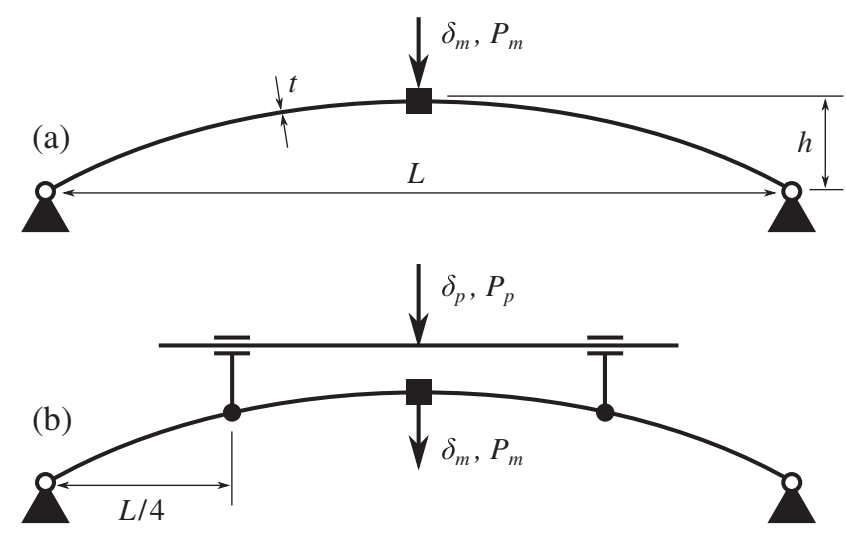

FIG. 2. (a) The shallow arch structure studied in this Letter. The edges of the arch are pinned. A vertical displacement $\left(\delta_{m}\right)$ or force $\left(P_{m}\right)$ is applied to the midpoint. Rotations and lateral translations are constrained at the midpoint to preserve symmetry. (b) Additional control points provide shape control. A displacement $\left(\delta_{p}\right)$ or force $\left(P_{p}\right)$ is applied symmetrically to the "probes" halfway between the midpoint and edges. The probes allow rotations and lateral displacements in order to prevent reaction moments and horizontal reaction forces that would force the structure into a different equilibrium.

inaccessible segments of equilibrium manifolds—but also the insight for a solution. For loops to exist, the structure must exhibit multiple different values of midpoint force for one midpoint displacement, and vice versa. By examination of the various arch shapes in Fig. 3(c) and corresponding force values, it is evident that each force corresponds to a unique arch shape. Hence, each combination of midpoint force, midpoint displacement, and deformation shape corresponds to a unique equilibrium.
Control over the deformation shape is the key ingredient for decoupling force and displacement at the control point. For example, for a given midpoint displacement, the reaction force at the same point can be controlled indirectly by changing the deformation shape.

The idea of separating force and displacement at a control point through shape control provides the first fundamental building block towards experimental continuation. The method for shape control is shown in Fig. 2(b). Namely, we add two (to enforce symmetry) probe points, which allow us to manipulate the arch shape using another displacement-controlled input.

Experimental continuation requires feedback control interlinking the loading and shape. Herein, we propose a simpler experiment as an initial step towards the full capability. Rather than moving the midpoint and probes simultaneously, we fix the midpoint at a given displacement and move the probes to scan for other equilibria. When the force on the probes reads zero, an equilibrium state of the system is found. With this method, which was recently used to determine localized solutions of the axially compressed cylinder [5,6], we find unstable equilibria which have never before been pinpointed quasistatically. This concept of obtaining a zero-force reading on the probe to determine equilibria is analogous to the minimization of virtual work in response to a probing virtual displacement, the vanishing of the residual in Newton's method at a converged state, and zero control in dynamic experimental continuation [2].

In previous work, the existence of unstable static equilibria has been intuited dynamically in the transients induced by large perturbations [7]. Our approach differs in that the experimental setup stabilizes otherwise unstable
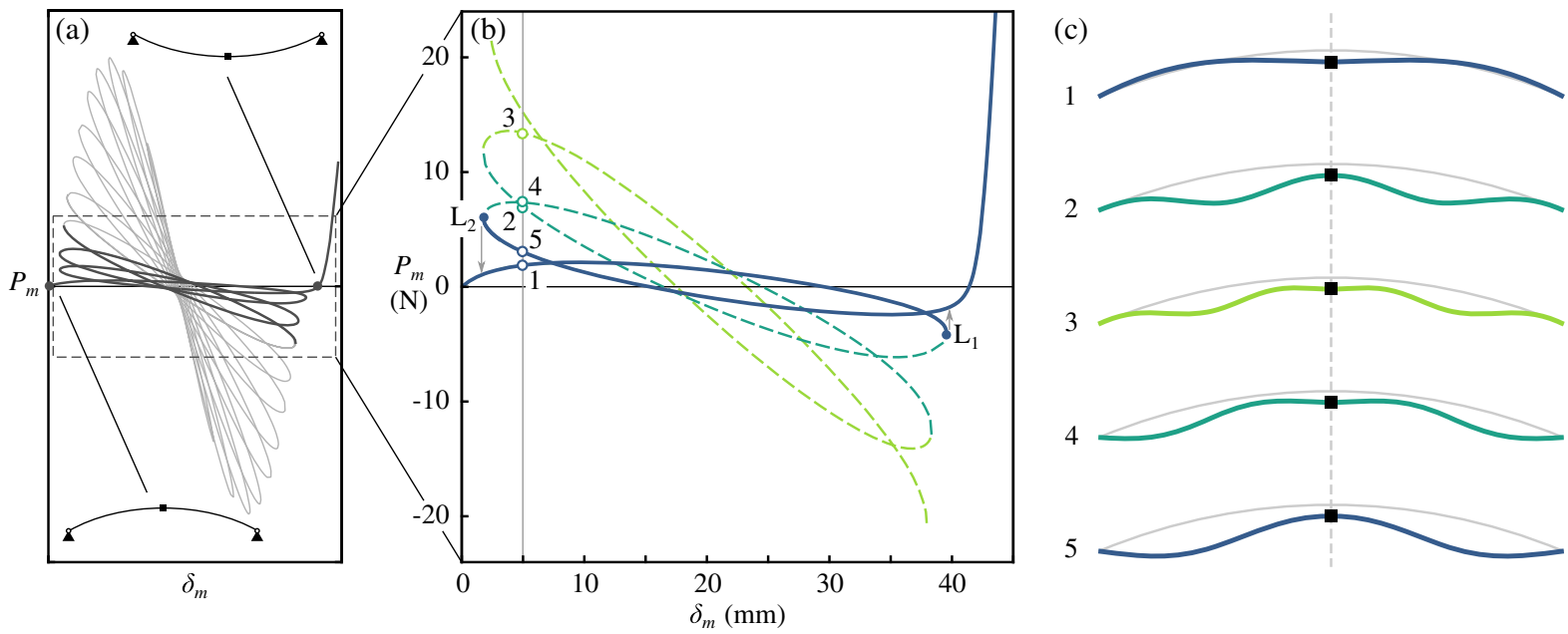

FIG. 3. FE prediction of the highly nonlinear load-displacement behavior of the shallow arch. (a) The entirety of the symmetric response. The arch shapes corresponding to the first and final equilibria are shown. (b) A subset of the response, with the solid blue lines indicating the segments a displacement-controlled experiment would obtain; dashed lines indicate the equilibrium configuration currently inaccessible experimentally. At the limit points $L_{1}$ and $L_{2}$, displacement control snaps to the opposite blue segment of the equilibrium curve. At $\delta_{m}=5$, there exist multiple load values for a given midpoint displacement (points 1-5). (c) The arch shapes at points $1-5$ for a fixed midpoint displacement. 

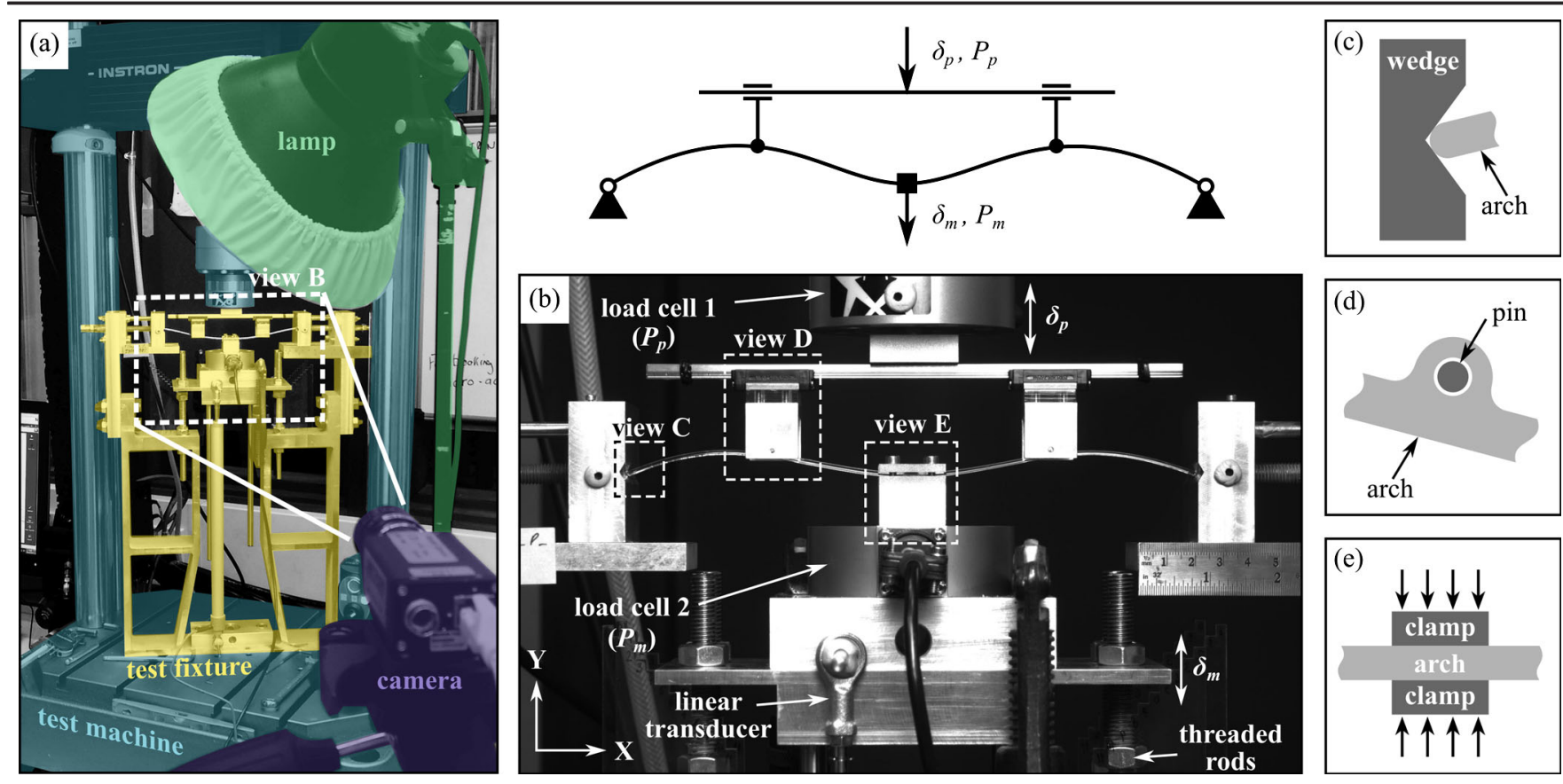

FIG. 4. Experimental setup. (a) The test fixture supporting the arch is bolted to the base of the test machine. The dashed rectangle shows the camera's field of view. (b) Physical implementation of the arch loads and boundary conditions. The idealized model is shown above for reference. The test machine controls the probe displacement $\delta_{p}$, and load cell 1 measures the probe force $P_{p}$. A movable platform sets the midpoint displacement $\delta_{m}$. A linear transducer measures the position of the midpoint platform. Load cell 2 measures the midpoint force $P_{m}$. (c) Wedge-shaped blocks restrain translations and allow rotations of the arch ends. (d) A linear guide rail allows the probes to move in the $X$ direction while controlling the displacement along $Y$. The probes connect to the arch with pins through builtin loops on the arch, which allow rotations. The loops were designed to be as small and noninvasive as possible. (e) A clamp restrains all translations and rotations at the arch midpoint to maintain symmetry. The clamped area is $5 \mathrm{~mm}$ wide.

equilibria using additional control points. Additional control points have been used to constrain [8] or probe [6] nonlinear structures in one direction but are here rigidly fixed to the structure to allow the push-pull control required to scan for additional equilibria.

Experimental methods.-Figure 4 gives a detailed explanation of the experimental setup, including the implementation of the loads and boundary conditions.

An Instron 8872 hydraulic test machine with an Instron Dynacell $\pm 250 \mathrm{~N}$ tension-compression dynamic load cell (load cell 1) was used for all displacement-controlled tests. Load cell 2 was a $\pm 500 \mathrm{~N}$ tension-compression load cell manufactured by Applied Measurements Ltd. A Gefran PZ34-A-250 linear transducer was used to measure the height of the midpoint platform. LabView (version 14.0) was used to log experimental data to ensure that synchronized readings were obtained from the various sensors. An Imetrum Video Gauge camera system was used to record the tests.

The geometry of the arches tested is shown in Fig. 2, with dimensions $L=205 \mathrm{~mm}, h=20 \mathrm{~mm}, t=1.57 \mathrm{~mm}$, and depth $D=4.68 \mathrm{~mm}$ (into the page). Ten specimens were manufactured using a Trotec Speedy 100 laser engraver to cut the arches from sheets of acrylic (supplied by F. R. Warren Ltd). Mechanical coupon testing was performed to find the Young's modulus $E=3200 \pm$ $70 \mathrm{MPa}$ and Poisson's ratio $\nu=0.38 \pm 0.02$ for use as inputs to finite element (FE) models.

Two types of tests were performed: midpoint scans and probe scans (videos are provided in Supplemental Material [9]). Midpoint scans represent the standard displacementcontrolled experimental approach. The test machine is connected to the midpoint clamp, and no probes are used. The midpoint is moved down and back up under displacement control. This produces a load-displacement curve similar to the solid blue lines in Fig. 3(b). The two segments of the equilibrium curve correspond to the "downwards" and "upwards" parts of the test. At limit points $L_{1}$ and $L_{2}$, the arch snaps to the other blue segment as indicated by the arrows. A midpoint scan was performed for each of the 10 arch specimens.

In a probe scan, the configuration in Fig. 4(b) is used. The midpoint is fixed at a given displacement, and the probes are moved down and back up under displacement control. During this test, the arch passes through both stable equilibrium segments and one or more unstable segments, as depicted in Fig. 5.

By repeating the probe scans at different midpoint displacements, the location of additional unstable equilibrium segments can be "mapped out" without path following. For 


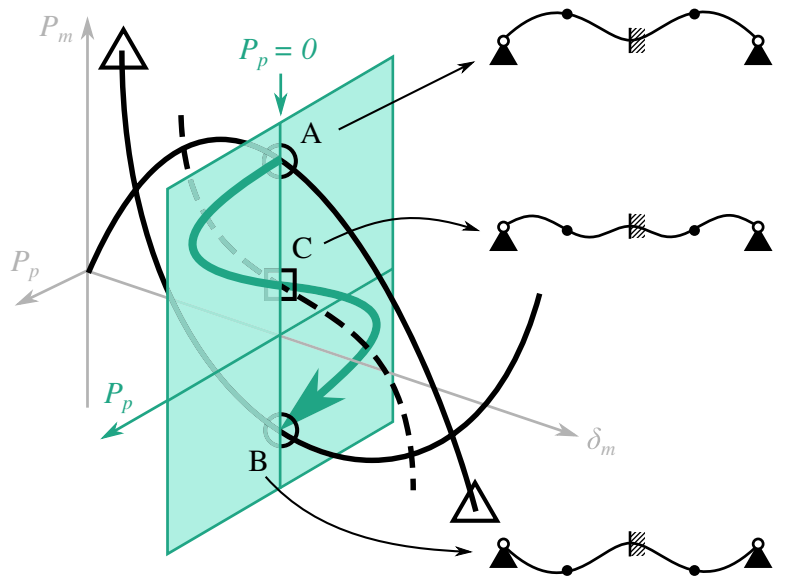

FIG. 5. A schematic midpoint scan is plotted in black on the $P_{m}-\delta_{m}$ plane. The probe scan takes place on the green $P_{p}-P_{m}$ plane, which intersects the midpoint scan at a fixed value of $\delta_{m}$. Starting at shape $\mathbf{A}$, the probes move down to shape $\mathbf{B}$. The probe reaction force $P_{p}$ is plotted on the green plane. For $P_{p}=0$, an equilibrium configuration is detected. The probe scan detects the segments of the equilibrium curve which are stable with midpoint control only (A and B) and also detects an unstable segment $(\mathbf{C})$.

each arch specimen, a probe scan was performed for $\delta_{m}=$ $\{8,12,16,20,24,28,32\} \mathrm{mm}$.

It is important to note that this method does not require us to follow or balance on an unstable equilibrium path; the structure is simply pushed through an unstable equilibrium, and its location on the midpoint load-displacement curve is measured. Consequently, the probe scan experiment can be performed by a displacement-controlled test machine. In future experiments, the probes will be controlled (via a more sophisticated feedback-control approach) to seek zero reaction force and follow an unstable equilibrium segment while moving the midpoint.

Results.-The midpoint scan $\delta_{m}, P_{m}$ data from 10 specimens were split into the downwards and upwards portions of the test, to prevent the loops in the plot affecting the following calculations. The data were then separated into $1 \mathrm{~mm}$ wide bins, and the mean and standard deviation of $P_{m}$ was found for each bin. Figure 6(a) shows the results in purple and blue. The height of the filled area indicates the confidence interval of the measurements, based on the standard deviation. For a more detailed discussion of the processing of experimental results, we refer the reader to Supplemental Material [9].

The probe scan $\delta_{p}, P_{p}$ data were analyzed to find all the zero crossings of $P_{p}$-i.e., the equilibria of the midpointcontrolled structure. The pairs of data points where $P_{p}$ crossed zero were identified, and the $\delta_{p}$ values corresponding to $P_{p}=0$ were found by linear interpolation. The gradient of the load-displacement curve and the shape of the arch at the equilibrium point revealed the segment to which the equilibrium belonged.

Two unstable segments were detected, in addition to the two known stable segments [Fig. 6(a)]. In all probe scans, one unstable equilibrium was found in the first (downwards) part of the test, and the other was found in the second (upwards) part of the test. Consequently, equilibria
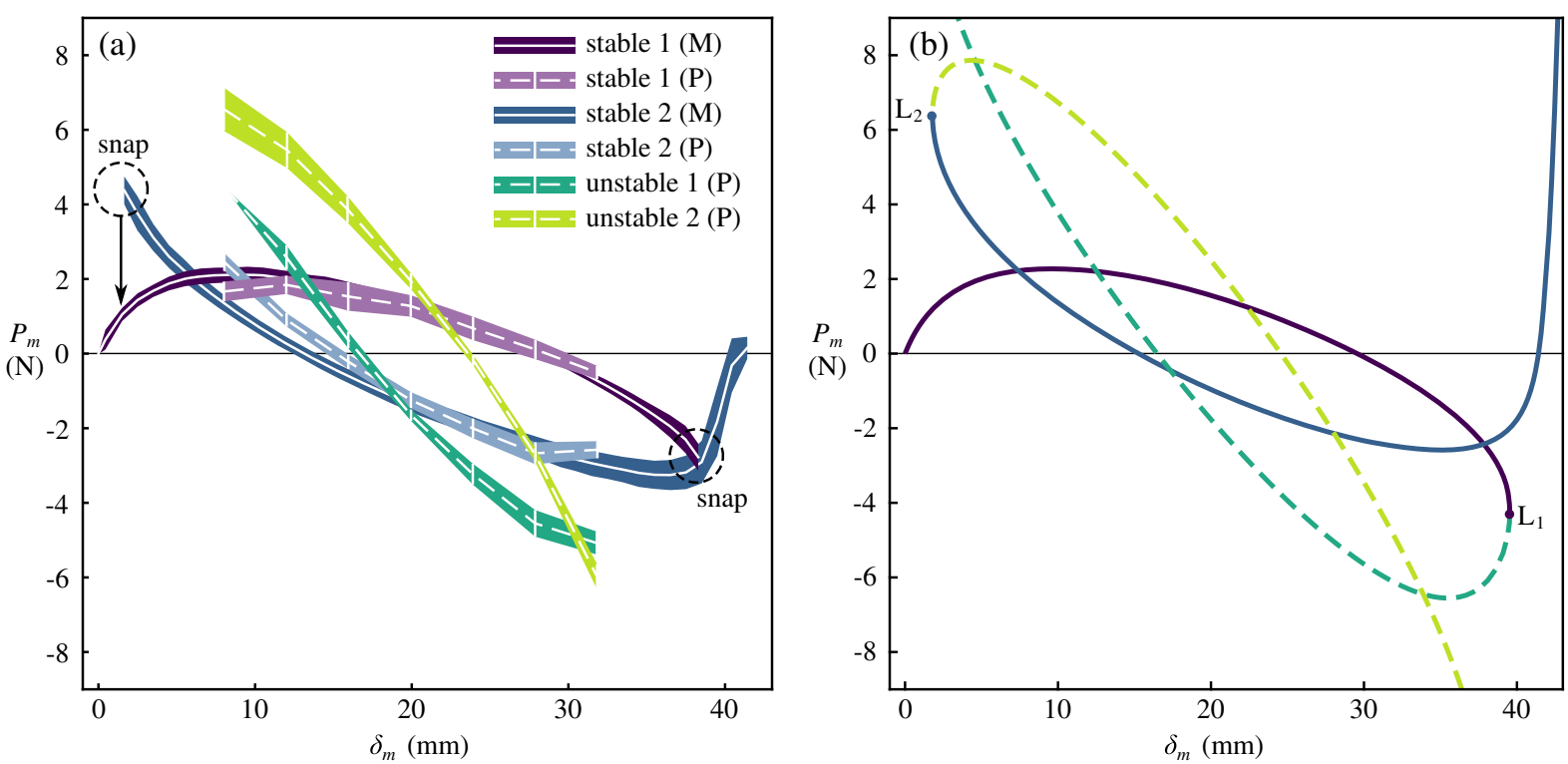

FIG. 6. Comparison of experimental and FE results. (a) Midpoint scan and probe scan results. Midpoint data after the snaps have been omitted. The white lines show the mean of the data, and the colored areas represent \pm one standard deviation of $P_{m}$. The midpoint scans are indicated by an " $M$ " in the legend; the probe scans by a " $P$." The vertical white bars show the $\delta_{m}$ locations of the probe scans. (b) FE prediction of the midpoint symmetric load-displacement response, truncated to the "first" few segments as in Fig. 3(b). The segments are color coded to match their counterpart in the experimental results. 
corresponding to the downwards or upwards part of the test are labeled with a 1 and a 2 , respectively.

At each $\delta_{m}$ location, the equilibria detected by the probe scans were grouped by type, and the mean and standard deviation of $P_{m}$ were calculated for each group. The aggregate data for each equilibrium type were then connected to give the mean \pm standard deviation plot shown in Fig. 6(a). The results of the probe scans match the results of the midpoint scans fairly well, despite the fact that the two types of tests are performed separately and with different equipment setups. Consequently, it is assumed that the unstable segments have been located with a similar degree of accuracy.

Discrepancies between the probe and midpoint scan results may be due to relaxation of the specimen material. Upon removing the arch from the fixture after the final probe scan, it was noted that most specimens did not immediately spring back to their undeformed shape. Imperfections in the experimental setup (especially the boundary conditions) may also have contributed to the differences.

An FE analysis of the arch was performed using nonlinear beam elements and idealized pinned boundary conditions. Figure 6(b) shows the FE prediction, with the segments color coded to match their experimental counterparts in Fig. 6(a). This shows that we have located the "next" two segments of the arch response-i.e., the segments beyond limit points $L_{1}$ and $L_{2}$. These segments correspond to arch shapes which are not stable under midpoint control only [shapes 2 and 4 in Fig. 3(c)] but are stable when supported by the probes. Further segments will correspond to more complex shapes [e.g., shape 3 in Fig. 3(c)], which will require additional probes for support.

Despite the sensitivity to initial conditions in nonlinear systems, there is excellent qualitative and quantitative agreement between the experimental and theoretical results. This provides confidence that the unstable equilibria have been correctly identified by the testing method.

Conclusions and outlook.-We have presented an experimental method to detect and identify unstable equilibria of nonlinear structures quasistatically, using probes to control the shape of the structure. Using this method, we have, for the first time experimentally, shown the location of unstable equilibria of a shallow arch which would not be accessible using traditional quasistatic testing techniques. These equilibria correspond to structural shapes that have zero reaction force at the probe points. The shape control provides independent, albeit indirect, control over the force and displacement at the point of actuation, which are otherwise intrinsically linked.

The natural extension of this work is to exploit the probing technique as a means to trace equilibrium paths of nonlinear structures. This may be achieved through a concerted variation in the actuation point force or displacement and shape control via the probes. The result is an experimental continuation technique, which enables the quasistatic nonlinear response of a structure to be explored systematically. In the future, the addition of multiple, independent probes would provide more refined control over the structural shape, thereby enabling more segments of the equilibrium manifold to be identified experimentally.

The development of continuation techniques using shape control will enable the experimental validation of the response of nonlinear structures. In turn, this will help encourage the exploitation of nonlinear structures in engineering applications, for example, in morphing structures and compliant mechanisms.

Data are available at the University of Bristol data repository [10].

This work was funded by the Engineering and Physical Sciences Research Council (EPSRC) through Grants No. EP/N509619/1, No. EP/M013170/1, and No. EP/ P511298/1, and the Royal Academy of Engineering through the research fellowship scheme No. RFI201718 117178. The support of all funders is gratefully acknowledged. The authors also acknowledge the help of the University of Bristol technicians: G. Pearn, A. Kraft, R. Billingham, R. Bragg, and R. Hooper.

*alberto.pirrera@bristol.ac.uk

[1] E. Riks, An incremental approach to the solution of snapping and buckling problems, Int. J. Solids Struct. 15, 529 (1979).

[2] J. Sieber, A. Gonzalez-Buelga, S. A. Neild, D. J. Wagg, and B. Krauskopf, Experimental Continuation of Periodic Orbits through a Fold, Phys. Rev. Lett. 100, 244101 (2008).

[3] H. Harrison, Post-buckling behaviour of elastic circular arches, Proc. Inst. Civil Eng. 65, 283 (1978).

[4] J. M. T. Thompson, Instabilities of elastic and spinning systems: Concepts and phenomena, Int. J. Bifurcation Chaos Appl. Sci. Eng. 27, 1730029 (2017).

[5] J. Thompson, J. Hutchinson, and J. Sieber, Probing shells against buckling: A nondestructive technique for laboratory testing, Int. J. Bifurcation Chaos Appl. Sci. Eng. 27, 1730048 (2017).

[6] E. Virot, T. Kreilos, T. M. Schneider, and S. M. Rubinstein, Stability Landscape of Shell Buckling, Phys. Rev. Lett. 119, 224101 (2017).

[7] R. Wiebe and L. N. Virgin, On the experimental identification of unstable static equilibria, Proc. R. Soc. A 472, 20160172 (2016).

[8] P. Harvey and L. Virgin, Coexisting equilibria and stability of a shallow arch: Unilateral displacement-control experiments and theory, Int. J. Solids Struct. 54, 1 (2015).

[9] See Supplemental Material at http://link.aps.org/ supplemental/10.1103/PhysRevLett.120.254101 for videos of the experiments and detailed explanation of data processing.

[10] See https://doi.org/10.5523/bris.2czhnqivx8i452gchxu104ur7q. 